\title{
Hysteresis of Switching Waves and Dissipative Solitons in Nonlinear Magnetic Metamaterials
}

\author{
N. N. Rosanov ${ }^{a, b}$, N. V. Vysotina ${ }^{a, b}$, A. N. Shatsev ${ }^{a, b}$, I. V. Shadrivov ${ }^{a, c}$, and Yu. S. Kivshar ${ }^{a, c}$ \\ ${ }^{a}$ St. Petersburg State University of Information Technologies, Mechanics, and Optics, St. Petersburg, 197101 Russia \\ e-mail: nrosanov@yahoo.com \\ ${ }^{b}$ Vavilov State Optical Institute, Birzhevaya liniya 12, St. Petersburg, 199034 Russia \\ ${ }^{c}$ Nonlinear Physics Center, Australian National University, Canberra ACT 0200, Australia
}

Received April 20, 2011

\begin{abstract}
Localized structures forming in the bistable regimes in a chain of weakly coupled split ring resonators, which are the building blocks of a nonlinear magnetic metamaterial, where electric current is generated by external electromagnetic radiation, have been studied analytically and numerically. The hysteresis of the velocity of switching waves (fronts) has been revealed and discrete dissipative solitons have been found.
\end{abstract}

DOI: $10.1134 / \mathrm{S} 0021364011120125$

Artificial composite structures containing electric conducting elements or metamaterials have recently attracted much attention in view of their unique properties of negative magnetic susceptibility and backward wave propagation. In contrast to crystals, metamaterials allow control of the macroscopic characteristics by choosing the types and geometry of their structural elements [1-3]. Resonant magnetic metamaterials are most simply obtained by creating a periodic lattice of resonant electric circuits that are much smaller than the wavelength of propagating electromagnetic waves. The artificial character of metamaterials makes it possible to control their properties through either the dynamic rearrangement of their structure or the inclusion of additional nonlinear elements and control their properties by an external field [4].

It has recently been shown that magnetic metamaterials consisting of chains of electric oscillatory circuits - split ring resonators - can exhibit discreteness effects owing to their weak coupling [5-8]. In particular, it was demonstrated that local nonlinearity and weak coupling between nonlinear split ring resonators in the chain (one-dimensional discrete system, where each split ring resonator interacts with nearest neighbors, can lead to the formation of discrete localized structures $[7,8]$. In this work, we study two types of discrete localized structures in such systems, namely, switching waves and dissipative solitons. We show that switching waves are immobile or move depending on the parameters of the system and prehistory (initial conditions), whereas solitons are highly localized due to the discreteness of the system.

Following [6], we consider a periodic chain of identical nonlinear split ring resonators (see Fig. 1), which is a simple one-dimensional model of a mag- netic metamaterial recently created and studied experimentally [9]. All split ring resonators lie in one plane and their centers are located on a straight line. Each split ring resonator can be associated with a nonlinear electric oscillatory circuit consisting of a nonlinear ohmic resistance, inductance, and capacitance. We assume that the nonlinearity of split ring resonators is caused by the Kerr nonlinearity of a medium introduced into the gaps of split ring resonators $[4,6]$.

The master equation for the amplitude of the electric current $\Psi_{n}$ in the $n$th resonator of the chain has the dimensionless form [6]

$$
\begin{aligned}
& i \frac{\partial \Psi_{n}}{\partial t}-\left(2 \Omega-i \gamma+\alpha\left|\Psi_{n}\right|^{2}\right) \Psi_{n}-\Sigma \\
& \quad=\kappa\left(\Psi_{n+1}+\Psi_{n-1}-2 \Psi_{n}\right) .
\end{aligned}
$$

Here, $t$ is the time divided by the period of natural oscillations of an isolated resonator; $\Omega$ and $\gamma$ is the deviation of the eigenfrequency from the frequency of pump radiation and the current decay rate in the resonator, divided by the eigenfrequency of the resonator; $\alpha= \pm 1$ and -1 for the self-focusing and self-defocusing nonlinearity, respectively; $\Sigma$ is the pump radiation amplitude; and $\kappa$ is the coupling constant. The slowly

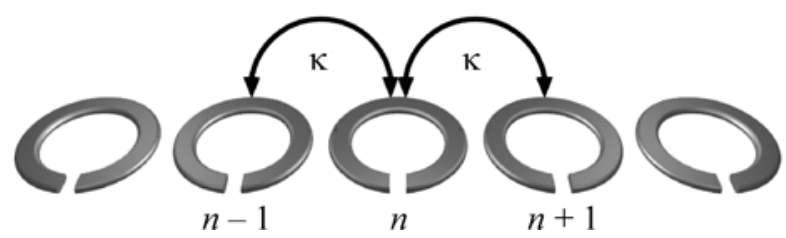

Fig. 1. Chain of split ring resonators weakly coupled with nearest neighbors. 


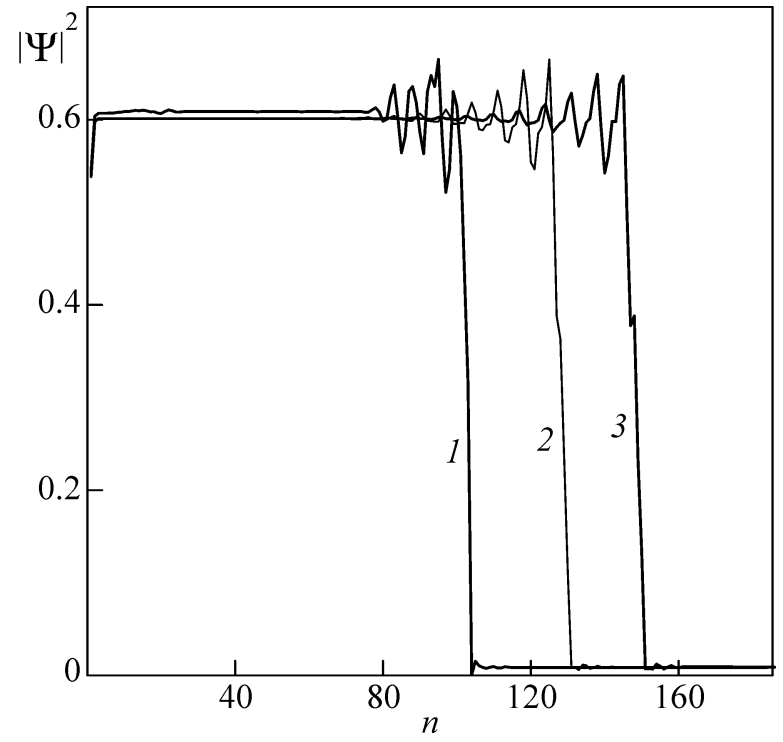

Fig. 2. Profiles of the moving switching wave at times $t=$ (1) 600 , (2) 1400 , and (3) $2000 ; \Sigma=0.05, \Omega=-0.2, \gamma=$ 0.02 , and $\kappa=0.07$.

varying amplitude approximation and nearest neighbor interaction approximation are used.

The continuous limit corresponds to large coupling constants. In this case, Eq. (1) coincides with the equation for an interferometer with a Kerr medium excited by external radiation $[10,11]$. An additional factor is the possible existence of not only positive, but also negative $\kappa$ values (the right-hand side of Eq. (1) presents discrete diffraction). The limit of vanishing coupling constants $\kappa \longrightarrow 0$ is degenerate (completely independent resonators). Under the conditions $\Omega^{2}>$ $3 \gamma^{2}$ and $\alpha \Omega<0$, bistability occurs: one of two current values $\Psi_{n}^{(0)}=\Psi^{(\uparrow)}$ and $\Psi^{(\downarrow)}$ is established depending on the initial conditions. In the case of weak coupling, perturbation theory in $\kappa$ is applicable. Setting $\Psi_{n}=$ $\Psi_{n}^{(0)}\left(1+\kappa \delta \Psi_{n}\right)$, we obtain the following equation for the corrections $\delta \Psi_{n}$ in the stationary regime:

$$
a_{n} \delta \Psi_{n}+b_{n} \delta \Psi_{n}^{*}=c_{n},
$$

where

$$
\begin{gathered}
b_{n}=\alpha\left|\Psi_{n}^{(0)}\right|^{2} \Psi_{n}^{(0)}, \\
a_{n}=b_{n}-\Sigma \Psi_{n}^{(0)}, \\
c_{n}=-\kappa\left(\Psi_{n+1}^{(0)}+\Psi_{n-1}^{(0)}-2 \Psi_{n}^{(0)}\right) .
\end{gathered}
$$

The solution of Eq. (2) has the form

$$
\delta \Psi_{n}=\frac{c_{n} a_{n}^{*}-b_{n} c_{n}^{*}}{\left|a_{n}\right|^{2}-\left|b_{n}\right|^{2}} .
$$

If the control parameter, which is pump radiation amplitude $\Sigma$, is smoothly varied, the denominator in Eq. (4) is zero at values corresponding to the edges of the intermediate (unstable) branch of the dependence of $\left|\Psi^{(0)}\right|^{2}$ on $\Sigma$. For this reason, the corrections can be large only near the edges of the lower and upper branches of this dependence.

The modulation instability of uniform distributions $\Psi_{n}^{(0)}=\Psi^{(0)}$ was studied in [6]. New results of numerical calculations of discrete switching waves and discrete dissipative solitons in the case of self-focusing nonlinearity $\alpha=1$ are reported below.

Switching waves. Under bistability conditions, when two stable uniform distributions exist in the chain, switching waves can form; the front of these waves constitutes a transition from one uniform distribution to another with a change in resonator number $n$ and uniform external excitation (the asymptotic values of the current amplitude $\Psi^{(\downarrow)}$ and $\Psi^{(\uparrow)}$ in the limits $n \longrightarrow \pm \infty$ are different). Only immobile switching waves are stationary. Their profiles are close to a step with corrections given by Eq. (4). In view of the discreteness of the system, the velocities and profiles of moving switching waves vary quasiperiodically with time (in contrast to a switching wave with a stationary profile in continual schemes). For this reason, the velocity of the switching wave $v$ is treated as its average value. The velocity is considered positive if the motion of the switching wave leads to the expansion of the region occupied by the upper branch of bistability (current amplitude $\left.\Psi^{(\uparrow)}\right)$; otherwise, the velocity is treated as negative. Figure 2 exemplifies the switching wave with positive velocity.

Since the system is discrete, the velocity of the switching wave is zero in a certain $\Sigma$ range. The $v(\Sigma)$ dependence exhibits hysteresis (see Fig. 3). For the accepted parameters, with an increase in $\Sigma$, immobile switching waves near the right edge of their existence range are smoothly transformed to moving switching waves (supercritical bifurcation). Near the left edge of the range, subcritical bifurcation occurs with a stepwise change in velocity. The bistability range of switching waves, where both immobile and moving switching waves exist, which is numerically determined with the parameters used in Fig. 3, is $0.0271 \leq \Sigma \leq 0.03135$.

The existence of a pump radiation amplitude range in which the switching wave is immobile is a feature of not only discrete, but also inhomogeneous continual systems [11] whose limiting case is the discrete system under consideration. For homogeneous continual systems, this range collapses to a point, which is the Maxwell value of the control parameter. Even under conditions when solitons do not form (see below), the properties of discrete switching waves lead to spatial hysteresis features [11] (pump radiation in the form of a beam whose amplitude varies slowly with time), 


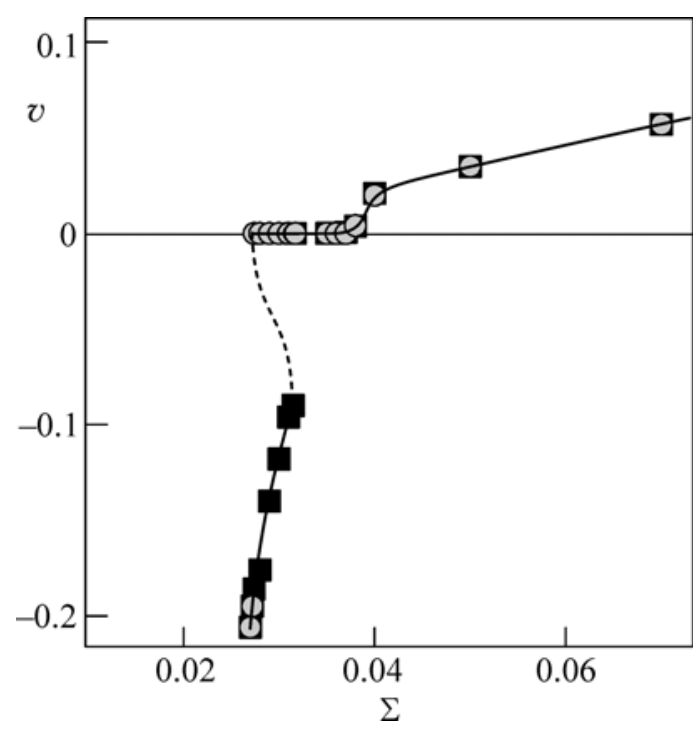

Fig. 3. Velocity of the switching wave $v$ versus the amplitude of pump radiation $\Sigma$. The arrows indicate hysteresis jumps under smooth variation of $\Sigma$. The parameters are $\Omega=-0.2, \gamma=0.035$, and $\kappa=0.089$.

which is similar to hysteresis in the case of ferromagnetism.

Discrete dissipative solitons. For light solitons (with a local increase in the current amplitude), the asymptotic values of the current amplitude in the limits $n \longrightarrow$ $-\infty$ and $n \longrightarrow+\infty$ coincide with $\Psi^{(\downarrow)}$. The relation $\Psi_{n}^{(0)}=\Psi^{(\downarrow)}+\left(\Psi^{(\uparrow)}-\Psi^{(\downarrow)}\right) \delta_{n, n_{0}}$ is valid for the narrowest soliton in the lowest approximation in $\kappa$. Corrections given by Eq. (4) are significant for a few neighboring resonators and are in agreement with the numerical calculations confirming the stability of soliton regimes (see Fig. 4). As is seen in Fig. 5, solitons are stable almost throughout the bistability range. For a sufficiently long chain, there are numerous solitontype profiles with various numbers of current maxima of various widths.

Thus, immobile and moving switching waves and discrete dissipative solitons exist in a chain of weakly coupled split ring resonators, which is essentially a nonlinear magnetic metamaterial excited by monochromatic electromagnetic radiation under bistability conditions. The corresponding electric current distributions are efficiently described by perturbation theory in the coupling constant. Hysteresis of switching waves has been revealed when either an immobile or moving switching waves appear depending on the initial conditions at given parameters of the system. This hysteresis can be attributed to the difference between static and kinetic friction in a discrete system (in a mechanics analogy, a force acting on a body is insufficient to overcome static friction, but can maintain the existing motion of the body). The existence of switching waves and dissipative solitons indicates the possi-

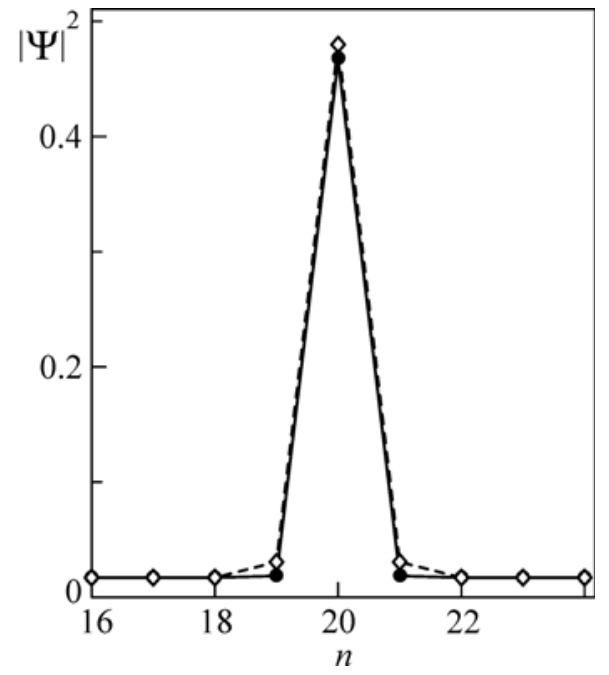

Fig. 4. Profile of the soliton at rest obtained in the (solid line) numerical calculation and (dashed line) perturbative calculation. The parameters are $\Omega=-0.2, \gamma=0.04, \kappa=$ 0.06 , and $\Sigma=0.04$.

bility of the formation of diverse structures and their dynamic rearrangement in a metamaterial irradiated by external radiation.

These results indicate that experimental investigations of discrete switching waves and dissipative solitons in a nonlinear metamaterial that were studied in $[12,13]$ in optical and microwave ranges are promising. We emphasize that the hysteresis of the velocity of switching waves is fundamentally different from the

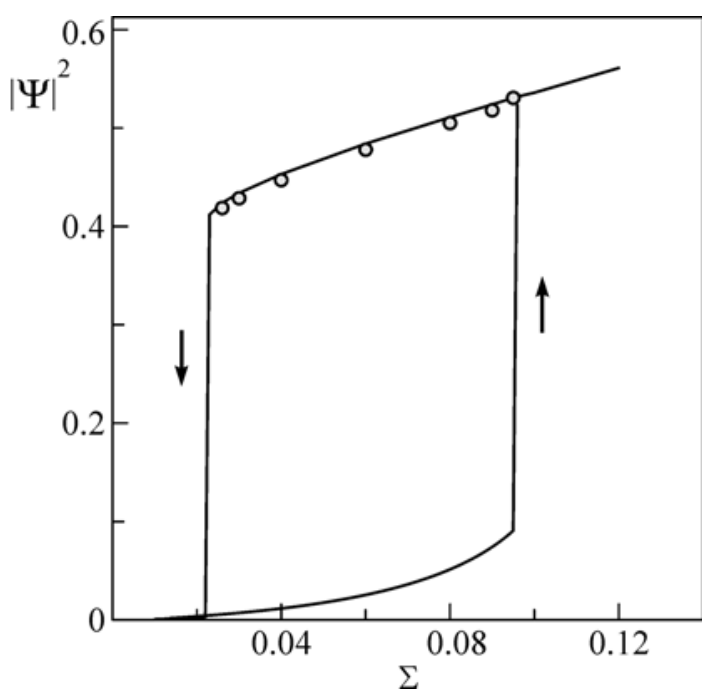

Fig. 5. Branches of the bistable response. Switching between them is shown by vertical arrows, circles near the upper branch indicate $\left|\Psi_{n}\right|^{2}$ values in the center of the narrowest soliton. The parameters are $\Omega=-0.2, \gamma=0.035$, and $\kappa=0.0025$. 
classical hysteresis of the amplitude of a nonlinear oscillator. The effects predicted in this work can be used to control the response of metamaterials in the nonlinear regime.

This work was supported by the Government of the Russian Federation (project for state support of research headed by leading scientists in Russian higher professional education institutions) and the Ministry of Education and Science of the Russian Federation (project no. RNP 2.1.1/9824).

\section{REFERENCES}

1. D. R. Smith, W. J. Padilla, D. C. Vier, et al., Phys. Rev. Lett. 84, 4184 (2000).

2. J. B. Pendry, Phys. World 14, 47 (2001).

3. A. V. Kil'dyshev and V. M. Shalaev, Phys. Usp. 54, 53 (2011).

4. A. A. Zharov, I. V. Shadrivov, and Y. S. Kivshar, Phys. Rev. Lett. 91, 037401 (2003).

5. E. Shamonina, V. A. Kalinin, K. H. Ringhofer, and L. Solymar, J. Appl. Phys. 92, 6252 (2002).
6. I. V. Shadrivov, A. A. Zharov, N. A. Zharova, and Y. S. Kivshar, Photon. Nanostruct. Fundam. Appl. 4, 69 (2006).

7. N. Lazarides, M. Eleftheriou, and G. P. Tsironis, Phys. Rev. Lett. 97, 157406 (2006).

8. Nonlinearities in Periodic Structures and Metamaterials, Ed. by C. Denz, S. Flach, and Yu. S. Kivshar (Springer, Heidelberg, 2010).

9. I. V. Shadrivov, A. B. Kozyrev, D. W. van der Weide, and Y. S. Kivshar, Opt. Express 16, 20266 (2008).

10. L. A. Lugiato and R. Lefever, Phys. Rev. Lett. 58, 2209 (1987).

11. N. N. Rosanov, Spatial Hysteresis and Optical Patterns (Springer, Heidelberg, 2002).

12. G. Dolling, M. Wegener, A. Schadle, et al., Appl. Phys. Lett. 89, 231118 (2006).

13. A. B. Kozyrev, C. Qin, I. V. Shadrivov, et al., Opt. Express 15, 11714 (2007).

Translated by $R$. Tyapaev 